\title{
NONLINEAR IONIC TRANSPORT THROUGH MICROSTRUCTURED SOLID ELECTROLYTES: HOMOGENIZATION ESTIMATES
}

\author{
Ignacio J. Curto Sillamoni and Martín I. Idiart \\ Departamento de Aeronáutica, Facultad de Ingeniería, Universidad Nacional de La Plata, Avda. 1 esq. \\ 47 S/N, La Plata B1900TAG, Argentina \\ Consejo Nacional de Investigaciones Cientificas y Técnicas (CONICET), CCT-La Plata, Calle $8 N^{o}$ \\ 1467, La Plata, B1904CMC, Argentina.
}

Keywords: diffusion, migration, heterogeneous solids, periodic homogenization, nonlinearity

\begin{abstract}
The most promising solid electrolytes available to date consist of poly-ethylene-oxide (PEO) complexes doped with a lithium salt such as lithium perchlorate $(\mathrm{LiClO} 4)$ or lithium iodide (LiI). At room temperature, the transport of lithium ions through these electrolytes is believed to occur by a hopping mechanism whereby, under the action of an electric field, ions jump along coordination sites available throughout the polymeric chains. Under moderate electric field intensities this mechanism results in a weakly nonlinear relation between the current density of electric charge and the electric field intensity which for most practical purposes can be approximated by a linear relation. Above a certain field intensity, however, a sudden increase of electric current is observed and the response becomes strongly nonlinear. The range of electric field intensities delimited by this threshold is known as the electrochemical stability window of the electrolyte and bears relevance to battery design for it dictates the electrochemical compatibility between electrodes and the electrolyte. Now, this window can vary consirably with microstructural properties such as crystallinity and second-phase inclusions. The purpose of this work is to put forward a multi-scale approach to estimate such variations. To that end, we consider the transport of multiple ionic species by diffusion and migration through microstructured solid electrolytes in the presence of strong electric fields. The assumed constitutive relations for the constituent phases follow from convex energy and dissipation potentials which guarantee thermodynamic consistency. The effective response is heuristically deduced from a multi-scale convergence analysis of the relevant field equations. The resulting homogenized response involves an effective dissipation potential per species. Each potential is mathematically akin to that of a standard nonlinear heterogeneous conductor. A 'linearcomparison' homogenization technique is then used to generate multi-scale estimates for these nonlinear potentials in terms of available estimates for corresponding linear conductors. By way of example, use is made of the Maxwell-Garnett and Effective-Medium linear approximations to generate estimates for two-phase systems with power-law dissipation. Explicit formulas are given for some limiting cases. In the case of threshold-type behavior, the estimates exhibit non-analytical dilute limits and seem to be consistent with fields localized in low energy paths. The estimates should be relevant for assessing the electrochemical stability of microstructured solid electrolytes.
\end{abstract}

